SANDRA BLAS-YAÑEZ Doctora en Ciencias Agropecuarias y Recursos Naturales por la Universidad Autónoma del Estado de México (UAEM) Profersora e investigadora de la Universidad Politécnica de Atlacomulco México [s.blasyanez@gmail.com]

HUMBERTO THOMÉ-ORTIZ Doctor en Ciencias Agrarias por la Universidad Autónoma Chapingo (México) Profesor e investigador del Instituto de Ciencias Agropecuarias y Rurales de la Universidad Autónoma del Estado de México

México

[humbertothome@hotmail.com]

ANGÉLICA ESPINOZA-ORTEGA Doctora en Ciencias de la Producción y la Salud Animal por la Universidad Nacional Autónoma de México (UNAM) Investigadora del Instituto de Investigación en Ciencias Agropecuarias y Rurales (ICAR) de la Universidad Autónoma del Estado de México Coordinadora de la Red Temática-CONACYT, Sistemas Agroalimentarios Localizados (REDSIAL)

México

[angelica.cihuatl@gmail.com]

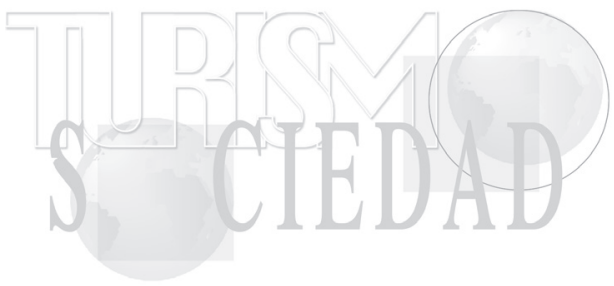

IVONNE VIZCARRA-BORDI Doctora en Antropología por la Universidad Nacional Autónoma de México (UNAM) Miembro honorable de la Asociación Mexicana de Estudios Rurales (AMER) Líder de la Red Maíz: Alimentación, Tecnología, Ecología y Cultura

Asesora de instancias gubernamentales en equidad de género Creadora del programa universitario de Estudios de Género y profesora de posgrados en Ciencias Agropecuarias Recursos Naturales, Ciencias de la Salud, Derecho, Ciencias Jurídicas y en Políticas Públicas en la Universidad Autónoma del Estado de México (UAEM) México [ivbordi@hotmail.com]

\section{TURISMO \\ AGROALIMENTARIO Y \\ BIENES DE CAPITAL: EL CASO DE LOS PRODUCTORES DE \\ PULQUE EN EL ALTIPLANO CENTRAL MEXICANO1}

AGRICULTURAL TOURISM AND

CAPITAL PROPERTY: THE CASE

OF PULQUE PRODUCERS

IN THE MEXICAN CENTRAL

HIGHLANDS

\section{Resumen}

Desde una perspectiva bourdesiana, se analizan las estrategias de acumulación y conversión de distintas formas de capital (cultural, económico, simbólico y social) que los productores de pulque ponen en práctica en los procesos de construcción social de iniciativas de turismo agroalimentario. Se trata de una nueva actividad rural a la que los productores se incorporan con desventajas materiales y culturales respecto a las reglas del campo turístico consensuadas por los actores dominantes. A partir de un enfoque cualitativo y exploratorio, se estudió la Feria del Pulque en Jiquipilco (Estado de México), en la que se identificó que la apropiación turística del patrimonio agroalimentario se consolida por medio de la reproducción continua de normas, valores y estrategias diferenciados entre los agentes que se disputan los beneficios de la actividad turística.

1 Para citar el artículo: Blas-Yañez, S., Thomé-Ortiz, H., Espinoza-Ortega, A. y Vizcarra-Bordi, I. (2020). Turismo agroalimentario y bienes de capital: el caso de los productores de pulque en el altiplano central mexicano. Turismo y Sociedad, XXVII, pp. 127-143.

DOI: https://doi.org/10.18601/01207555.n27.07

Fecha de recepción: 26 de marzo de 2019

Fecha de modificación: 11 de junio de 2019

Fecha de aceptación: 27 de agosto de 2019 
Palabras clave: Turismo agroalimentario, bienes de capital, pulque, campo cultural.

\begin{abstract}
From a Bourdieu critical perspective, the strategies of accumulation and exchange of different forms of capital (cultural, economic, symbolic and social) that pulque producers wield in the processes of social construction of agri-food tourism initiatives are analyzed. It is a new rural activity to which producers are incorporated with material and cultural disadvantages resulting from the rules of the tourist field agreed upon by dominant actors. Through a qualitative and exploratory approach, the pulque fair was studied in the state of Jiquipilco, Mexico, where it was identified that the tourist appropriation of the agri-food heritage is consolidated through the continuous reproduction of norms, values and differentiated strategies among the agents disputing the benefits derived from this tourism activity.
\end{abstract}

Keywords: Agri-food tourism, capital goods, pulque, cultural arena.

\section{Introducción}

En la actualidad, algunos alimentos tradicionales anteriormente clasificados como "comida de pobres" - como es el caso de los insectos 2 , la chía o el pulque ${ }^{3}$ - son

2 La ingesta de insectos comestibles en México se asocia con las etnias originarias del territorio nacional, dado el amplio conocimiento y aprovechamiento de la biota local que los nativos - y aun mucha gente - de áreas rurales utiliza para autoconsumo y comercialización. Entre los órdenes de insectos más consumidos en México se tienen los siguientes: Hymenoptera, Hemiptera, Coleoptera, Orthoptera y Lepidoptera (Ramos-Elorduy y Viejo Montesinos, 2007).

3 Bebida de origen prehispánico, de bajo contenido alcohólico, producto de la fermentación de savia fresca (aguamiel) que se extrae de varias especies de agave (maguey) que crecen en el centro de México. Durante el apogeo de la cultura azteca, se producía y consumía en rituales sagrados; después de la Conquista, el pulque perdió gradualmente su valor sagrado y revalorizados en el mercado bajo el precepto de desarrollo local (Suremain y Matta, 2013). Lo anterior se logra por medio de prácticas discursivas fundadas en investigación médica, agroecológica y disposiciones patrimoniales que reclasifican estos alimentos como productos "nutracéuticos, súper alimentos, auténticos, naturales o justos" (García, 2010; Guerrero et al., 2009).

Los alimentos emblemáticos con identidad territorial son un componente central de las industrias culturales y recreativas contemporáneas, tal y como lo son el turismo rural y el agroalimentario (Matta, 2019), actividades que se rigen por la lógica de la economía de la calidad ligada al territorio. Lo anterior se traduce en una puesta en valor que simultáneamente es una acción política atravesada por relaciones de poder y procesos de institucionalización en la que convergen una heterogeneidad de agentes, intereses y relaciones sociales (Del Mármol, Siniscalchi y Estrada, 2016).

En este sentido, se considera que los efectos del turismo son un factor de cambio que influye en el entorno social a partir del ordenamiento de los grupos sociales (Liang y Chan, 2018), de la fragmentación del capital social (Ramírez, Cruz, Vargas y Rodríguez, 2019) y de las adaptaciones en la estructura y en la cultura tradicional (George y Reid, 2005). Es este el motivo por el que la institucionalización de las políticas de revalorización agroalimentaria por medio del turismo produce cambios en el entorno local y propicia una nueva clasificación de los grupos sociales en el campo del turismo (Liang y Chan, 2018).

Es importante hacer un análisis reflexivo sobre el desarrollo de iniciativas de turismo

\footnotetext{
se convirtió en una bebida alimenticia y embriagante popular asociada con la cultura alimentaria indígena (Escalante, López, Velázquez, Giles-Gómez, Bolívar y López-Munguía, 2016).
} 
agroalimentario en entornos rurales y enfatizar en las estrategias que los actores locales despliegan para mantenerse dentro del espacio relacional que representa el turismo agroalimentario.

El objetivo de este estudio fue documentar los mecanismos de acumulación y conversión de bienes de capital que los productores de pulque ponen en práctica para permanecer y competir en la actividad turística emergente.

Las teorías del capital y del campo cultural de Bourdieu $(1993 ;$ 1985) se retomaron para comprender los procesos de acumulación y conversión de bienes de capital que despliegan los productores rurales para ingresar y posicionarse en la actividad turística que surge en la localidad.

El texto se divide en cinco partes: seguido de este apartado introductorio, se describe el marco teórico y conceptual que rige la discusión del documento; posteriormente, se presenta el diseño metodológico del caso estudiado; más adelante, se describen las formas de acumulación y conversión de bienes de capital que posicionan y permiten el reenclasamiento de los productores rurales en el emprendimiento turístico; para finalizar, el texto concluye con una reflexión acerca de los principales hallazgos de la investigación.

\section{Turismo agroalimentario como subcampo del patrimonio cultural}

"Campo cultural" es un concepto repensado por Pierre Bourdieu (1985) para referirse a un espacio social estructurado por instituciones, reglas, rituales, convenciones, categorías y discursos que producen una lógica, normas y valores inherentes a la producción y al consumo de la cultura (Schmitz, Witte y Gengnagel, 2017).

Un campo se estructura por agentes que dominan y por agentes dominados (Bourdieu,
1998), resultado de un tejido de relaciones y posiciones sujetas a la concentración de capitales específicos mediante los cuales las estructuras de poder son movilizadas (Schmitz et al., 2017). En dicho espacio social, el capital (económico, social, cultural y simbólico) es el medio para competir por una posición dominante (Çakmak, Lie y McCabe, 2018b), por lo cual, los agentes entran en conflicto y competencia al tratar de acumular, conservar o convertir diferentes tipos de capital (Ihlen, 2007).

Las cuatro grandes formas de capital que Bourdieu distingue y sugiere como principios de construcción del espacio social son las siguientes: i) capital social, capacidad de movilizar recursos por medio de redes y vínculos sociales; ii) capital económico, factores de producción y riqueza acumulada; iii) capital cultural en tres formas: a) incorporado (características, habilidades y capacidades mentales y físicas que se debilitan y mueren con su portador), b) institucionalizado (títulos académicos) y c) objetivado (bienes culturales materiales, como trofeos, instrumentos, muebles); y iv) capital simbólico, una forma intangible de representar simbólicamente cualquiera de las otras formas de capital (Bourdieu y Wacquant, 1992; Bourdieu, 1993).

Los capitales fundan la dialéctica del desclasamiento y del reenclasamiento a partir del principio de reconversión de Bourdieu, que permite a los poseedores de determinado capital mantener su posición en la estructura social por medio de la reconversión de las formas de capital que ellos poseen en otras formas más rentables y legítimas en el campo adscrito (Bourdieu, 2011).

La teoría del campo de producción cultural de Bourdieu se aplica al ámbito del patrimonio cultural alimentario de diversas maneras (Matta, 2019), puesto que el patrimonio agroalimentario es un elemento de afirmación 
territorial (Suremain, 2017) fundado en discursos e instituciones globales ${ }^{4}$ que norman y regulan formas de protección y promoción de culturas alimentarias en peligro de extinción y la expansión continua de las industrias culturales (Matta, 2019).

El turismo agroalimentario es considerado un subcampo del patrimonio cultural, dado que constituye un sistema de disposiciones, agentes y financiamientos que, desde la perspectiva de las nuevas configuraciones territoriales de la ruralidad, sirven para la conformación de este tipo de turismo. Dicha actividad económica se concibe como un nuevo escenario de disputa de bienes de capital que se inscribe en el campo socioeconómico del patrimonio cultural. Es por ello por lo que el turismo es analizado como un escenario rural de posiciones materiales y subjetivas donde existen diversidad de agentes, roles y capitales que cada actor posee, capital simbólico que le es reconocido socialmente.

El subcampo del patrimonio agroalimentario se basa en discursos esencialistas (BlasYañez, Thomé-Ortiz, Espinoza-Ortega y Vizcarra-Bordi, 2018a) en los que se valoran capitales asociados con la identidad territorial, la autenticidad, el terruño, la naturaleza y la tradición (Espeitx, 1996). Esto ha generado

4 En el campo del patrimonio cultural, uno de los agentes dominantes es la Organización de las Naciones Unidas para la Educación, la Ciencia y la Cultura (Unesco), que encabeza las acciones de valorización del patrimonio agroalimentario a nivel mundial. Las reglas o doxa se fundan en diversas declaraciones que imprimen las pautas de valorización de sistemas agroalimentarios, como son i) la Recomendación sobre la Salvaguarda de la Cultura Tradicional y Popular, de 1989, que buscó disminuir los riesgos de la homogeneización alimentaria resultado de la globalización, industrialización y urbanización avasallante (Sureiman, 2017); ii) la Convención sobre Patrimonio Cultural Inmaterial de la Humanidad, realizada en 2003, que abrió la posibilidad de incluir la alimentación como patrimonio cultural (Amaya, 2013); y iii) la Lista Representativa del Patrimonio Cultural Inmaterial de la Humanidad, en la que la diversidad de alimentos y formas de producción agroalimentaria se perfila en los expedientes de este organismo para salvaguardar su reproducción material y simbólica (Unesco, 2003). un fenómeno global a partir del cual las grandes metrópolis y provincias de América Latina han desplegado diversas formas de valorización alimentaria (Suremain, 2017), tales como ferias y rutas agroalimentarias, marcas colectivas, indicaciones geográficas, sellos de calidad y denominaciones de origen, que se vinculan directamente al turismo, al desarrollo y al sector agroindustrial (Espeitx, 2004; Bessière, 1998).

Estas prácticas de valorización territorial de alimentos integran el conocimiento de alimentos tradicionales con preocupaciones socioeconómicas y ambientales, con principios éticos y salud pública, para reagrupar los alimentos marginados con base en cánones altos y cosmopolitas, lo que lleva a la "gentrificación de los alimentos nativos" (Matta, 2019). De esta manera, los productores rurales de productos primarios se convierten en proveedores de bienes culturales. Así, su identidad, su saber-hacer y su cultura sirven de medios para la obtención del mejor rendimiento del capital cultural que poseen, con lo que producen bienes particulares $\mathrm{y}$, gracias a ello, consiguen beneficios de diversa índole (Bourdieu, 1985).

\section{Metodología}

Se desarrolló un estudio de caso simple (Stake, 2000), de tipo cualitativo y exploratorio, en un período comprendido entre 2015 y 2018, en Jiquipilco, un municipio del centro de México (figura 1) con arraigada tradición pulquera, que desde el año 2010 ha incursionado en la revalorización de esta bebida popular como estrategia de desarrollo rural.

Se consideró la puesta en marcha de la Feria del Pulque de Jiquipilco como un ejemplo de "microcosmos" de relaciones y posiciones sociales (Ahmad, 2013) donde se mantiene una lógica de revalorización agroalimentaria centrada, en este caso, en 


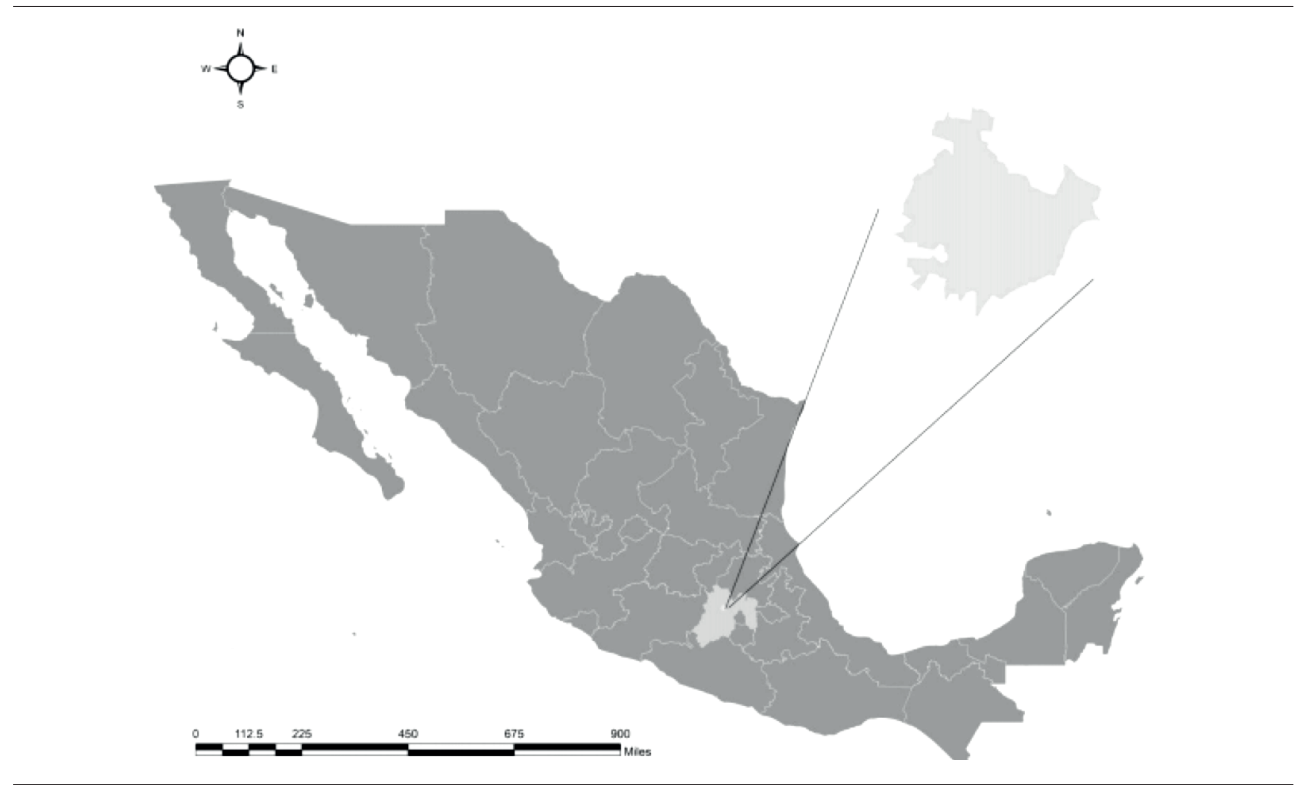

Fuente: INEGI (2016).

una bebida ancestral con arraigo territorial. Este espacio físico y relacional se rige por normas específicas de acceso, participación, derechos, obligaciones y sanciones, y donde los capitales de cada agente determinan su posición dentro del espacio social construido.

Se utilizó un muestreo intencional (Çakmak, Lie y Selwyn, 2018a) para capturar una variedad de perspectivas sobre las diferentes formas de capital de los agentes involucrados en la expresión turística. Con la finalidad de obtener mayores conocimientos sobre el fenómeno de la iniciativa turística, mirándola desde varios ángulos (Çakmak et al., 2018a), se entrevistó a dos servidores públicos que dirigen y promueven la feria, a dos estudiantes que realizan trabajo de investigación en el municipio y a diez productores que participaron en la feria durante el tiempo de observación.

Se realizaron entrevistas semiestructuradas y se llevó a cabo la observación directa del fenómeno, que fue anotada en el diario de campo. Las entrevistas estuvieron compuestas por preguntas principales, seguidas de interrogantes detalladas sobre los siguientes aspectos: i) roles de los actores, ii) capitales desplegados y iii) conflictos asociados con el desarrollo del turismo agroalimentario.

Las entrevistas y las notas tomadas en el diario de campo fueron analizadas con base en el método de análisis de contenido (Hsieh y Shannon, 2005; Piñuel, 2002). Para el análisis de la información, se realizó un abordaje relacional a partir de tres dimensiones propuestas por Ahmad (2013): i) análisis de las condiciones sociales, de los instrumentos políticos y de los discursos que estructuran el campo; ii) mapeo del funcionamiento del espacio social construido en términos de lo que se valora en ese escenario; y iii) análisis de las estrategias de acumulación y conversión de capitales entre los agentes que participan en la práctica turística.

Primero se registraron y resumieron las respuestas textuales de los entrevistados, 
también se generó una base de interpretaciones axiales sobre las variables analizadas (capital económico, social, cultural y simbólico), con lo cual se logró clasificar en subgrupos a los productores participantes. Posteriormente, se compararon las opiniones de los grupos entrevistados con el propósito de identificar los capitales reconocidos socialmente en cada subgrupo y diferenciar sus formas de acumulación y conversión de capitales para ingresar, permanecer y posicionarse en el desarrollo turístico emergente.

\section{Resultados y discusión}

\section{Potencial del capital cultural de la producción de pulque como estrategia de desarrollo territorial}

El pulque que se produce en el centro de México es una bebida popular reconocida y valorada por sus consumidores rurales y urbanos; es comercializada directamente por sus productores en mercados cercanos a las localidades rurales que la elaboran (Blas-Yañez, Thomé-Ortiz, Vizcarra-Bordi y Espinoza-Ortega, 2018b).

Esta bebida se produce en pequeña escala en el contexto del trabajo familiar de los productores. El saber-hacer de este oficio se desarrolla a partir de aprendizajes adquiridos en la infancia del productor y de la acumulación de experiencia en el tiempo, y se convierte en capital cultural incorporado. Este capital descansa en los productores de mayor edad; su transmisión es oral y práctica entre generaciones en el seno familiar, y corre el riesgo de desaparecer por ser un capital incorporado que se debilita y muere con el portador, tal como sucede con otros productos agroalimentarios tradicionales, como la sal de Zapotitlán Salinas (Puebla) (Thomé-Ortiz, Renard-Hubert y De Jésus Contreras, 2017).
Para mantener y proteger este tipo de capital, han surgido una diversidad de discursos e instituciones que ven en el turismo un factor de crecimiento económico y un elemento que contribuye a conservar la naturaleza y la diversidad cultural desde lo local (Marín, García y Daltabuit, 2012). Por medio de habilitadores - como políticas institucionales de apoyo, consumidores innovadores y nuevo capital emprendedor (Mitchell y Shannon, 2018) — , se han impulsado cambios en los modelos de producción y en las actividades agropecuarias del medio rural (Matta, 2019); ello ha generado la emergencia de procesos de patrimonialización de recursos agroalimentarios locales a partir de la inserción del turismo en los sistemas de producción local.

Por esta razón, en el ámbito rural se observa una efervescencia de estrategias de valorización — como ferias y rutas agroalimentarias- que surgen como nuevos mercados de la cultura alimentaria marginal, cristalizada en la tercerización del agro mexicano. Esto implica la adaptación y apropiación de los espacios locales para generar, por medio de la diversificación económica de lo rural, nuevas pautas de producción y consumo de las formas de vida y la cultura local, modelos que imitan fielmente los procesos emergentes de la lógica capitalista y hedonista del consumo cultural.

\section{Conformación del microcampo de valorización recreativa del pulque}

La profundidad histórica 5 del pulque fue uno de los principales puntos de partida para la

\footnotetext{
5 Desde el siglo v a. C. hasta el siglo Xx d. C., diversas civilizaciones del centro de México han construido una cultura del maguey y tradición pulquera (Ramírez, 2007). Desde la época prehispánica, el pulque ha mantenido significados míticos y simbólicos asociados con la fertilidad, la ritualidad y la psicoterapia autóctona (Rivas, 2001; Dehouve, 2014 Mazzetto, Segura y Moragas, 2015). Cuando el Imperio azteca cayó, el pulque perdió su importancia religiosa, pero mantuvo su relevancia como suplemento nutricional y sustituto de agua (Lappe-Oliveras, Moreno-Terrazas, Arrizón-Gaviño,
} 
consolidación de una feria agroalimentaria. La estrategia se fundó en la instrumentalización del patrimonio local (Prats, 2005) para generar un emblema territorial capaz de favorecer el "desarrollo económico" (Álvarez y Medina, 2008; Acampora y Fonte, 2008).

La actividad turística en Jiquipilco implicó la movilización de actores internos y externos para consolidar la feria agroalimentaria como atractivo turístico. El gobierno local dirige y gestiona la Feria del Pulque en Jiquipilco desde el año 2010 como una nueva posibilidad productiva y de posicionamiento territorial, más allá de la producción agropecuaria que tradicionalmente define al municipio. Para ello fue necesaria la vinculación de la comunidad, los productores de pulque, las instituciones educativas y las instituciones gubernamentales locales y estatales.

La variedad de interacciones multiescala, dinámicas y juegos de diferentes formas de poder entre los actores involucrados genera la transformación del sistema agroalimentario (Rossi, Bui y Marsden, 2019) del pulque. Tal y como indican Thomé-Ortiz et al. (2017), la vinculación entre turismo y patrimonio agroalimentario se consolida por medio de redes entre actores locales y agentes externos para construir un producto turístico emblemático del territorio, fundado en el capital social y cultural.

Como indican Mitchell y Shannon (2018), el turismo en espacios rurales emerge a partir

\footnotetext{
Herrera-Suárez, García-Mendoza y Gschaedler-Mathis, 2008); entonces, por su alto contenido de vitaminas, era consumido por las mujeres en puerperio para estimular la producción de leche materna y recuperarse del parto; también hombres y niños lo bebían en el desayuno, la comida y durante la jornada de trabajo (Valadez, 2014). Entre los siglos XVII y XX, se consolidó una agroindustria alrededor del pulque, pero luego, a mediados del siglo XX, se abandonó y se desprestigió esta bebida como símbolo de ignorancia y subdesarrollo (Lutz, 2012). Actualmente, el pulque repunta en la investigación científica y en políticas públicas de desarrollo por sus propiedades nutricionales, ecológicas e identitarias y como patrimonio biocultural de las comunidades locales (Escalante et al., 2016).
}

de mecanismos de recombinación, conversión y estratificación de activos históricos asociados con actividades productivas que generan un escenario de desarrollo turístico. En este caso, la consolidación de la feria requirió de un producto con arraigo territorial identitario, además de diversas iniciativas, como la formación de grupos y la vinculación interinstitucional para fomentar la reconversión productiva estacional que representa la Feria del Pulque.

\section{Mapeo de agentes en el microcampo de la feria agroalimentaria}

Las ferias agroalimentarias han proliferado como una forma de atracción turística rural, actividad económica que desarrolla una relación específica con el espacio y la articulación entre diferentes agentes que intervienen en ella (Balslev y Velázquez, 2010). Estos agentes son actores individuales o colectivos que disponen de diferentes dotaciones y tipos de capital (Schmitz et al., 2017).

En la zona de estudio, el Gobierno local actúa como agente dominante, ejerce su poder al introducir mecanismos y reglas de organización específica para llevar a cabo la feria. Este agente exige a los productores de pulque mantener sus tradiciones, y se enfoca en revalorizar el maguey como planta y el pulque como bebida nativa del centro de México para recrear un "anacronismo patrimonial" que justifica la existencia de la feria (Suremain, 2017).

Así, la Feria del Pulque proyecta una identidad territorial que recupera las raíces prehispánicas de la producción de esta bebida - asociada con lo indígena y lo naturala partir de la estandarización del uso de madera para la construcción de módulos de exhibición y de contenedores de barro y jarros para la venta de pulque, con el fin de representar autenticidad a los ojos del consumidor contemporáneo (Espeitx, 2011). 
Esto expresa la manera como las políticas globales de desarrollo rural y las tendencias turísticas actuales se cristalizan en contextos específicos (Amaya, 2013) como estrategia de desarrollo local a partir de discursos esencialistas del patrimonio (Blas-Yañez et al., 2018a).

El gobierno local se enfoca en estrategias para el desarrollo económico por medio de la Feria del Pulque como atracción turística. Este agente acumula poder y dominación sobre el proyecto turístico por medio del capital económico que obtiene de la recaudación de impuestos y del capital social convertible en capital económico, proveniente de los vínculos que mantiene con instituciones estatales, como la Secretaría de Turismo (SECTUR) y la Secretaría de Economía (SE), que sirven para patrocinar parte de la infraestructura para la feria y la publicidad del evento. También, la vinculación con televisoras regionales y radiodifusoras ayuda a aumentar la publicidad local y regional de la feria, lo que se convierte en reconocimiento territorial del municipio y del producto con identidad local, es decir, en capital simbólico.

Como en el caso del turismo informal en destinos turísticos emergentes de Tailandia (Çakmak et al., 2018b), en Jiquipilco también se observa que las instituciones gubernamentales acumulan capital económico por medio de la recaudación de impuestos y se convierten en la autoridad decisiva para definir los valores del capital cultural que dan legitimidad a los participantes en la feria.

Las instituciones educativas, por su parte, mantienen el papel de agentes de cambio cultural o "intermediarios culturales" (Bourdieu,1984), actores con escaso capital económico y cierto capital cultural proveniente del sistema educativo, que ayudan a la socialización de nuevos estilos de vida asociados con el consumo de bienes simbólicos
(Moguillansky, 2008). Estos agentes sin fines de lucro se enfocan más en temas socioculturales, ambientales y de diversificación agroalimentaria para preservar el patrimonio material e inmaterial que sostiene el oficio de tlachiquero 6 . Las actividades de fortalecimiento de capacidades que desarrollan con los productores de pulque se dirigen a aumentar el capital cultural y social de la comunidad local.

Los productores de pulque, conscientes de que la naturaleza de su trabajo está cambiando, generan diferentes estrategias de acumulación y conversión de capitales para participar y posicionarse en la actividad turística emergente a partir de prácticas discursivas con el fin de hacer frente a la competencia extrema existente en los mercados de alimentos tradicionales.

\section{Bienes de capital y su conversión en la consolidación de expresiones de turismo agroalimentario}

En la actualidad, la construcción de identidades a partir de las actividades productivas tradicionales del campesino está adquiriendo un peso significativo en el ámbito rural, en la redefinición del territorio y de las clases sociales marginadas (Cowan y Schneider, 2008).

Las condiciones del mercado del consumo recreativo de la cultura alimentaria, los discursos patrimoniales y las demandas de los consumidores han fomentado la adaptación de estrategias, así como también la acumulación y conversión de capitales de los productores de pulque. De este modo, la convertibilidad de los diferentes tipos de capital

6 El término "tlachiquero" proviene del nahua tlachiqui, acción de raspar. Tlachiquero es la persona cuya actividad principal gira en torno del cultivo del maguey, con amplio conocimiento empírico y técnico para capar, raspar y recolectar el aguamiel diariamente (Ramírez, 2004; Rendón, 1990). 
es el mecanismo básico de las estrategias de reproducción social (Cowan y Schneider, 2008) de esta fracción de clase, con lo que busca que su negocio familiar sobreviva, alcance éxito y acumule capitales para obtener mayor poder en el entorno turístico y, con respecto a los agentes dominantes, estatus social y ganancias económicas.

Estos actores cuentan con un alto nivel de capital cultural en forma incorporada y objetivada, lo que facilita el acceso del patrimonio cultural al campo del turismo (Çakmak et al., 2018b). A este tenor, se identificaron tres tipos de productores de pulque que participan en la feria agroalimentaria: a) productores tradicionales, b) productores acaparadores y c) acaparadores ocasionales; las tres fracciones de clase cuentan con diferentes tipos de capitales (ver tabla 1).

La elasticidad de la definición técnica y social del oficio de pulquero ha permitido que agentes desligados del gremio pulquero hagan del turismo agroalimentario una posibilidad de inserción, dada la flexibilidad que caracteriza al sector turístico: intensiva mano de obra poco calificada, orientación a los servicios y generación de trabajos estacionales (Çakmak et al., 2018a). Sin embargo, la reinterpretación del pulque como recurso turístico ha generado tensiones entre los actores sociales y las formas en que se relacionan con el espacio y los recursos, tal y como lo indica el siguiente testimonio:

Ahora todos quieren ser productores de pulque. Los del municipio, con tal de ganar dinero, dejan que cualquiera entre a la feria a vender pulque, aunque sean rescatistas, y uno, que es productor y lleva producto de calidad, no nos dan nuestro lugar... si sin nosotros no hay feria (sic). (Testimonio, 2017).

Los actores que participan como productores de pulque en la feria tienen en común el aseguramiento del rendimiento máximo del

Tabla1. Tipos de productores de pulque

\begin{tabular}{|c|c|c|c|}
\hline $\begin{array}{l}\text { Trayectorias } y \\
\text { capitales }\end{array}$ & Productores tradicionales & Productores acaparadores & Acaparadores ocasionales \\
\hline Identidad & Continua & Reanudada & Adaptada \\
\hline Habitus & $\begin{array}{l}\text { Plantación y producción tra- } \\
\text { dicional } \\
\text { Comercio local }\end{array}$ & $\begin{array}{l}\text { Acaparamiento y comercia- } \\
\text { lización permanente en mer- } \\
\text { cados populares regionales }\end{array}$ & $\begin{array}{l}\text { Acaparamiento y comercialización } \\
\text { ocasional local y regional } \\
\text { Multiactividades productivas y de } \\
\text { servicios }\end{array}$ \\
\hline Capital social & Familia nuclear y extendida & $\begin{array}{l}\text { Familia nuclear, vecinos y } \\
\text { vendedores de otros gremios } \\
\text { inmersos en el comercio } \\
\text { regional }\end{array}$ & $\begin{array}{l}\text { Familia nuclear, vecinos y conoci- } \\
\text { dos local y regionalmente }\end{array}$ \\
\hline $\begin{array}{l}\text { Capital eco- } \\
\text { nómico }\end{array}$ & $\begin{array}{l}\text { Eslabón de producción de ma- } \\
\text { teria prima (aguamiel y pulque) } \\
\text { Comercialización a pie de } \\
\text { finca }\end{array}$ & $\begin{array}{l}\text { Eslabón de comercializa- } \\
\text { ción regional permanente } \\
\text { Distribución y valor agrega- } \\
\text { do del pulque }\end{array}$ & $\begin{array}{l}\text { Eslabón de comercialización local } \\
\text { y regional ocasional de acuerdo } \\
\text { con el calendario cívico-religioso } \\
\text { Distribución y valor agregado }\end{array}$ \\
\hline $\begin{array}{l}\text { Capital } \\
\text { cultural }\end{array}$ & Incorporado y objetivado & $\begin{array}{l}\text { Incorporado, institucionali- } \\
\text { zado y objetivado }\end{array}$ & Objetivado \\
\hline $\begin{array}{l}\text { Capital } \\
\text { simbólico }\end{array}$ & Legado familiar & Identidad territorial & $\begin{array}{l}\text { Mimetización de la identidad } \\
\text { territorial }\end{array}$ \\
\hline
\end{tabular}

Fuente: Elaboración propia con base en datos de campo. 
capital cultural según sus disposiciones, ya sea incorporado, institucionalizado u objetivado, directamente transmitido por la familia.

\section{a) Capital económico}

El grupo de productores tradicionales se caracteriza por poseer capital económico ligado al eslabón de producción, dado que cuentan con una superficie agrícola superior a dos hectáreas en donde mantienen plantíos de agave pulquero, y el único subproducto que obtienen es el aguamiel para producir pulque diariamente. En promedio, el volumen de producción diaria corresponde a raspar 7 20 magueyes $^{8}$ por persona dos veces al día, lo que representa un aproximado de $601 \mathrm{de}$ pulque al día. Su forma de comercialización es a pie de finca de manera convencional, y en festividades importantes, como el Día de Muertos o el Día de la Santa Cruz, se suele distribuir el pulque en panteones y santuarios, donde hay aglomeraciones sociales.

Los productores-acaparadores cuentan con un capital económico ligado al eslabón de comercialización del pulque y carecen, en su mayoría, de una superficie agrícola propia en donde tengan plantación de maguey. La forma de producción y recolección de aguamiel se hace por medio del capital social (con vecinos y familiares que tienen plantíos de maguey) de quienes acaparan diariamente entre 60 y 1001 de pulque por vendedor para ser comercializados fuera del municipio, dado que cuentan con los medios materiales y sociales para realizar la distribución permanente de pulque a regiones aledañas

\footnotetext{
7 Práctica de raspar el tejido del centro del maguey con una cuchara de metal para fomentar la producción de savia o aguamiel (Hinke, 1997).

8 Maguey es el nombre común del Agave spp., planta xerofita y suculenta que tiene hojas duras y alargadas con espinas en los bordes que crecen en forma de roseta. Esta planta se adapta a climas adversos, y se le atribuyen más de cien usos, entre ellos, en textiles, jabones, inulina, pulque, vinagre y destilados (Parra, Del Villar y Prieto, 2010).
}

al municipio, generalmente, mercados itinerantes y pulquerías.

En general, la solvencia de los productores de pulque se funda en el capital social que transforman en activo productivo, es decir, en el aguamiel y el pulque que acumulan a diario para vender a nivel local y regional, además de ser un activo principal durante la celebración de la feria agroalimentaria. Por lo tanto, el capital social es directamente convertible en capital económico por los productores locales entrevistados.

\section{b) Capital social}

El capital social de los productores de pulque reside principalmente en lazos de confianza en el interior de las familias y de la comunidad. Estos nexos de solidaridad se utilizan para acceder a capital económico, por ejemplo, para aumentar el volumen de producción de pulque en los días que se realiza la feria y para incrementar el capital cultural, principalmente objetivado e institucionalizado por medio de estrategias de inversión social colectiva.

La más sobresaliente fue la consolidación de una asociación de productores de agave pulquero en el año 2013, mediante la cual subvirtieron las limitantes de acceso y derecho a participar como "auténticos productores de pulque" en la feria agroalimentaria. El reconocimiento institucional de esta asociación ayudó a los agremiados a sobrevivir a la normativa de los agentes dominantes del campo, pues fue una acción propiamente política que generó ganancias simbólicas.

La institucionalización de la asociación sirvió como instrumento de defensa y reconocimiento del valor del trabajo que realizan los agremiados, y se mantiene como una forma reconocida de identidad colectiva, con lo que justifica plenamente su existencia. Así se informó en uno de los testimonios: 
Yo me acerqué a participar en el grupo para tener derecho de participar en la feria como productor que soy, porque a uno lo ven mal los del ayuntamiento, porque vendemos en los tianguis; ellos creen que no somos productores, pero sí lo somos, nada más que nosotros le buscamos para salir del mercado local. (Testimonio, 2018).

La conformación y el mantenimiento de la asociación los ha dotado del poder simbólico con el cual han logrado imponer reconocimiento social y político para demandar su derecho a participar en la feria agroalimentaria local anualmente.

La inversión en capital social colectivo permitió a los productores adquirir mayor conocimiento, establecer vínculos con agentes externos - como funcionarios o empresarios turísticos-y mantener mayor participación en eventos extralocales para ofertar su producto. En cuanto a la cantidad y estructura de su capital social, ahora es más fuerte e incide directamente en mejorar su posición social, simbólica y económica, lo que le hace posible al titular tener acceso a información y a recursos más sobresalientes que los que se obtienen de redes tradicionales (Çakmak et al., 2018a).

La cantidad de capital social aumenta con el uso (Çakmak et al., 2018a). La proliferación de ferias agroalimentarias representa un hecho estructural que permite que la actividad turística movilice vínculos comerciales extralocales para los productores de pulque, de manera que la feria es una plataforma de acumulación de capital social que posibilita y fomenta la heterogeneidad de las redes sociales.

\section{c) Capital cultural}

El capital cultural incorporado en forma de conocimiento sobre el oficio de tlachiquero y el saber-hacer del pulque es utilizado por los productores tradicionales como un activo competitivo único al comunicar el conocimiento y la experiencia adquiridos con el tiempo para operar y entregar una experiencia auténtica a los turistas y consolidar la identidad del alimento con el territorio.

El capital cultural institucionalizado es utilizado por los productores con trayectorias interrumpidas 9 en el oficio de pulquero para reagruparse como "auténticos productores de pulque" a partir de la adscripción a la asociación de productores y para mejorar la rentabilidad de la herencia cultural directamente transmitida por la familia (Bourdieu, 2011). Este grupo ha invertido capital económico y tiempo en cursos de capacitación e innovación agroalimentaria del agave pulquero que han gestionado con instituciones estatales y educativas, lo que implica la redefinición del valor del oficio y la reevaluación de sus oportunidades en el ámbito turístico. Esto es similar a lo reportado por Ahmad (2017) respecto a la lucha entre agencias de viajes, que se posicionan unas sobre otras por la acumulación de conocimiento acerca de lugares, comida, idiomas, etc.

Las tres fracciones de clases de productores identificadas utilizan el capital cultural objetivado - tales como fotografías antiguas, pieles de animales, artesanías de ixtle y herramientas tradicionales del oficio - para atraer a los clientes y convertir el capital cultural objetivado en capital económico por medio de habilidades, inmersión o medios de exhibición (Ellis, Park, Kim y Yeoman,

9 A este grupo se adhieren aquellos que provienen de una familia pulquera, actores individuales que durante su infancia y juventud temprana participaron en la producción y comercialización de pulque, pero que en algún momento de su vida se desvincularon del gremio para incorporarse a otras actividades económicas, y que posteriormente se han reincorporado a la producción y comercialización de pulque por representar una actividad que, de acuerdo con su experiencia, permite obtener un mayor margen de ganancias netas según las disposiciones forjadas en el núcleo familiar, es decir, un habitus ligado a la comercialización informal de pulque. 
2018). Esta mímesis ${ }^{10}$ (Bourdieu, 1990) de capital cultural objetivado ha aumentado y se ha venido unificando cada año en la feria, de esta forma se transfieren los significados institucionalizados sobre el valor, la nostalgia y el esencialismo patrimonial a la elaboración del "anacronismo patrimonial" (Suremain, 2017), y se saca el máximo rendimiento del capital cultural objetivado del oficio de tlachiquero.

Los productores de pulque, como en el caso de los empresarios de turismo informal, tienen un alto nivel de capital cultural y deben colaborar con los agentes hegemónicos para transformar el capital cultural en capital económico (Çakmak et al., 2018b). Por lo tanto, los productores de pulque mantienen una posición de subordinación debido a que están condicionados por factores estructurales y sociales de la economía de consumo.

\section{d) Capital simbólico}

La identidad referida a la producción de pulque se ha revalorizado a nivel local a partir de la conversión de capital cultural incorporado de los productores de antaño en capital simbólico. En función del reconocimiento social de aquellos que conservan de manera ininterrumpida las tierras donde crece el maguey y realizan las labores de producción de pulque como actividad principal de subsistencia por generaciones, estos agentes reciben mayor respeto por parte de los miembros de su comunidad como "auténticos productores de pulque 11 ".

\footnotetext{
10 Proceso de transferencia de significados institucionales a nivel microsocial a partir de la imitación inconsciente de prácticas de otros individuos, lo que permite la comprensión compartida de los significados asociados con la práctica (Bourdieu, 1990; Sieweke, 2014).

11 La reputación como cultivadores de maguey se reconoce a nivel local a partir del saber-hacer agrícola sobre el maguey pulquero, saber que es transmitido intergeneracionalmente en el interior de las familias. La especificidad del conocimiento biocultural que precisa el oficio de tlachiquero se materializa en la calidad y el manejo del pulque para mantener sus atributos
}

Los productores de pulque generan estrategias de conversión de sus bienes de capital para utilizarlos en expresiones de turismo ligadas al patrimonio cultural alimentario de la región. Por ser este capital apreciado por los visitantes, los productores tienden a buscar la ascensión de su estatus social y la construcción de capital simbólico como constantes en el desarrollo del turismo agroalimentario (Matta, 2016).

Dado que el capital simbólico abre nuevas vías en las redes sociales para obtener ventajas y mejor posición en múltiples campos (Çakmak et al., 2018a), los productores de pulque, como las cocineras tradicionales de Michoacán y Oaxaca (Matta, 2016), se han apropiado del patrimonio cultural material e inmaterial que representa el pulque para mejorar su estatus social (Liang y Chan, 2018) a partir del reenclasamiento que les proporciona el capital social y simbólico que han acumulado por medio de su participación en ferias regionales, nacionales y programas televisivos, que los reconocen como productores y protectores del patrimonio alimentario local.

La reconversión del sistema productivo del pulque está condicionada por las reglas de juego, en especial, las que imponen las instituciones públicas y el mercado. Sin embargo, estar en condiciones de cambiar dichas normas implica ser reconocido socialmente como un actor legítimo, como

\footnotetext{
de sabor, consistencia y grados de fermentación, que convergen con la construcción social de gusto. Normalmente, el oficio de tlachiquero viene acompañado de la posesión de las tierras de cultivo, la posesión de un tinacal (área de fermentación) y otra área para la venta de pulque. De acuerdo con lo anterior, en el presente trabajo se clasifica a los productores de pulque en tres categorías: i) productor tradicional o "auténtico" (realiza las tareas de todo el ciclo productivo, desde la plantación de agave, la extracción de aguamiel y la fermentación, hasta la comercialización a pie de finca), ii) acaparador permanente (acopia el producto local y lo distribuye en mercados regionales como fuente principal de ingresos durante todo el año) y iii) acaparador ocasional (rescata el producto local para revenderlo en festividades locales).
} 
"auténtico productor de pulque", lo que supone la acumulación de reconocimiento social o poder simbólico para participar e influir en las decisiones públicas, ocupar una posición privilegiada en la feria $\mathrm{y}$, de ese modo, crear el contexto más favorable para su reproducción social (Cowan y Schneider, 2008). Como indican Çakmak et al. (2018a), el capital cultural y el simbólico persisten como los más sobresalientes para los pequeños productores que buscan posicionarse en el entorno emergente de extensiones del turismo rural, como son las ferias agroalimentarias.

\section{Conclusiones}

La reestructuración económica del espacio rural se ha orientado a la inserción de nuevas actividades económicas, como el turismo. Por medio de la valorización de productos agroalimentarios con identidad territorial, los productores rurales generan nuevas estrategias económicas centradas en la lógica de acumulación y en la conversión de capitales, en las que el turismo juega un papel importante para garantizar la reproducción social de sistemas agropecuarios de subsistencia.

Los "nuevos intermediarios culturales" - en forma de políticas públicas, Estado y demás instituciones de investigación y servicios de consumo cultural — han ayudado a garantizar la supervivencia de formas de producción agroalimentaria marginales y han favorecido la reproducción social de los productores de pulque en condiciones de subordinación condicionada por factores estructurales y sociales de la economía de consumo.

Este estudio da cuenta de la complejidad inherente a la puesta en marcha de proyectos turísticos agroalimentarios en un entorno agropecuario, campesino y con baja capacitación sobre actividades terciarias. Así mismo, demuestra que los productores de alimentos tradicionales poseen un nivel notable de capitales culturales y sociales que les permite una inserción exitosa en el turismo del patrimonio cultural; sin embargo, los agentes públicos dominantes subestiman el valor de estos capitales. Por lo tanto, es evidente la importancia de generar programas de empoderamiento de los productores rurales para disminuir las desventajas competitivas en expresiones de turismo rural.

El aporte de la investigación radica en la aplicación de la propuesta analítica de Pierre Bourdieu para obtener una mejor comprensión de la inserción del turismo como estrategia de desarrollo y preservación de la cultura local en el ámbito rural. Esta perspectiva sirvió para examinar qué formas especializadas de capital se activan para construir expresiones turísticas en el medio rural, las formas en que son reproducidos los discursos patrimoniales globales a nivel local y los medios con los que diferentes grupos sociales negocian o imponen formas de reenclasamiento de oficios marginados.

Los resultados de este estudio exploratorio pueden ayudar a los formuladores de política turística municipal, regional y nacional a perfilar estrategias que consideren los efectos sociales del emprendimiento turístico en el ámbito rural y la continuidad de formas de alimentación tradicionales.

La limitación del estudio es que lo expresado no es generalizable a otras expresiones de turismo rural, dado que cada contexto y grupo social tiene diferentes capitales y formas de movilizarlos para la reconversión productiva rural.

Una investigación futura en el marco bourdesiano deberá identificar la relación entre los tres conceptos clave de esta teoría (capital, habitus y campo) en el desarrollo del turismo agroalimentario. Así mismo, aplicar una metodología cuantitativa ayudaría a generar una explicación más detallada sobre 
las disposiciones que permiten desarrollar estrategias diferenciadas de apropiación y reproducción del patrimonio agroalimentario.

\section{Referencias bibliográficas}

Acampora, T. y Fonte, M. (2008). Productos típicos, estrategias de desarrollo rural y conocimiento local. Revista Ópera, 7, 191-212. Recuperado de https://revistas.uexternado.edu.co/index.php/ opera/article/view/1199

Ahmad, R. (2013). Working with Pierre Bourdieu in the tourism field making a case for 'third world'. Tourism, Cultural Studies, 27(4), 519-539. DOI: 10.1080/09502386.2012.707222

Ahmad, R. (2017). Power struggles within and between organizations in tourism business: A bourdieusean approach to organizational analysis. Tourism Culture \& Communication, 17(1), 47-59. DOI: $10.3727 / 109830417 \mathrm{X} 14837314056898$

Álvarez, M. y Medina, X. (eds.). (2008). Identidades en el plato. El patrimonio cultural alimentario entre Europa y América. Barcelona: Icaria.

Amaya, S. (2013). Conflicto y poder entre actores sociales en los procesos de patrimonialización del jamón ibérico. Boletín de Antropología, 28(46), 100-123. Recuperado de http://aprendeenlinea. udea.edu.co/revistas/index.php/boletin/article/ view/19522

Balslev, H. y Velázquez, M. A. (2010). La posición social y espacial en una ciudad turística. Las luchas simbólicas de Álamos, Sonora. PASOS. Revista de Turismo y Patrimonio Cultural, 8(1), 47-59. DOI: $10.25145 /$ j.pasos.2010.08.004

Bessière, J. (1998). Local development and heritage: Traditional food and cuisine as tourist attractions in rural areas. Sociologia Ruralis, 38(1), 21-34. DOI: 10.1111/1467-9523.00061

Blas-Yañez, S., Thomé-Ortiz, H., EspinozaOrtega, A. y Vizcarra-Bordi, I. (2018a). La construcción discursiva del patrimonio agroalimentario en las sociedades contemporáneas: aproximaciones conceptuales y debates teóricos. Revista Mexicana de Ciencias Agrícolas, 9(2), 443-457. DOI: 10.29312/remexca.v9i2.1084

Blas-Yañez, S., Thomé-Ortiz, H., VizcarraBordi, I. y Espinoza-Ortega, A. (2018b). Street sale of pulque and socio spatial practices: A gender perspective in central Mexico. Journal of Ethnic Foods, 5(4), 311-316. DOI: 10.1016/j. jef.2018.10.005

Bourdieu, P. (1984). Distinction: A social critique of the judgment of taste. Cambridge: Harvard University Press.

Bourdieu, P. (1985). The social space and the genesis of groups. Theory and Society, 14(6), 723-744. DOI: $10.1007 / \mathrm{BF} 00174048$

Bourdieu, P. (1990). The logic of practice. Cambridge: Polity Press.

Bourdieu, P. (1993). The field of cultural production: Essays on art and literature. New York: Columbia University Press.

Bourdieu, P. (1998). Practical reason: On the theory of action. Palo Alto, CA: Stanford University Press.

Bourdieu, P. (2011). Las estrategias de la reproducción social (1. ${ }^{\mathrm{a}}$ ed.) (Trad. A. B. Gutiérrez). Buenos Aires: Siglo XXI Editores.

Bourdieu, P. y Wacquant, L. J. D. (1992). An invitation to reflexive sociology. Chicago: University of Chicago Press.

Çakmak, E., Lie, R. y Selwyn, T. (2018a). Informal tourism entrepreneurs' capital usage and conversion. Current Issues in Tourism, 22(18), 2.2502.265. DOI: $10.1080 / 13683500.2018 .1448763$

Çakmak, E., Lie, R. y McCabe, S. (2018b). Reframing informal tourism entrepreneurial 
practices: Capital and field relations structuring the informal tourism economy of Chiang Mai. Annals of Tourism Research, 72, 37-47. DOI: 10.1016/j.annals.2018.06.003

Cowan, C. y Schneider, S. (2008). Estrategias campesinas de reproducción social. El caso de las Tierras Altas Jujeñas, Argentina. Revista Internacional de Sociología, 66(50), 163-185. DOI: 10.3989/ris.2008.i50.100

Dehouve, D. (2014). Voy a cortar a una muchacha con mi gran cuchillo porque quiero beber un poco. La elaboración del pulque por los indígenas tlapanecos (México). Nuevo Mundo Mundos Nuevos [En línea]. DOI: 10.4000/nuevomundo.66731

Del Mármol, C., Siniscalchi, V. y Estrada, F. (2016). Reflecting on heritage and power: Dynamics, strategies and appropriations in the Catalan Pyrenees and the French Alps. International Journal of Heritage Studies, 22(5), 341-354. DOI: $10.1080 / 13527258.2016 .1153495$

Ellis, A., Park, E., Kim, S. y Yeoman, I. (2018). What is food tourism? Tourism Management, 68, 250-263. DOI: 10.1016/j.tourman.2018.03.025

Escalante, A., López Soto, D. R., Velázquez Gutiérrez, J. E., Giles-Gómez, M., Bolívar, F. y López-Munguía, A. (2016). Pulque, a traditional Mexican alcoholic fermented beverage: Historical, microbiological, and technical aspects. Frontiers in Microbiology, 7, 1.026. DOI: 10.3389/ fmicb.2016.01026

Espeitx, E. (1996). Los «nuevos consumidores» o las nuevas relaciones entre campo y ciudad a través de los «productos de la tierra». Agricultura y Sociedad, 80-81, 83-116. Recuperado de https:// www.mapa.gob.es/ministerio/pags/biblioteca/ revistas/pdf_ays/a080_04.pdf

Espeitx, E. (2004). Patrimonio alimentario y turismo: una relación singular. PASOS. Revista de Turismo y Patrimonio Cultural, 2(2), 193-213. DOI: $10.25145 /$ j.pasos.2004.02.016
Espeitx, E. (2011). Productos «de la tierra»y alimentos transgénicos: tradición e innovación como atributos valorados y como factores de riesgo. En E. Espeitx y J. Cáceres (eds.), Sabores culturales: ensayos sobre alimentación y cultura (pp. 118-158). Barcelona: Montesinos.

García, M. E. (2010). Super guinea pigs? Anthropology Now, 2(2), 22-32.

George, E. W. y Reid, D. G. (2005). The power of tourism: A metamorphosis of community culture. Journal of Tourism and Cultural Change, 3(2), 88-107. DOI: 10.1080/09669580508668489

Guerrero, L., Guàrdia, M. D., Xicola, J., Verbeke, W., Vanhonacker, F., Zakowska-Biemans, S. et al. (2009). Consumer-driven definition of traditional food products and innovation in traditional foods. A qualitative cross-cultural study. Appetite, 52(2), 345-354. DOI: 10.1016/j.appet.2008.11.008

Hinke, N. (1997). Breve léxico del maguey. Ciencias, 46, 26-29. Recuperado de http:// www.revistas.unam.mx/index.php/cns/article/ view/11560/10885

Hsieh, H-F. y Shannon, S. E. (2005). Three approaches to qualitative content analysis. Qualitative Health Research, 15(9), 1.277-1.288. DOI: $10.1177 / 1049732305276687$

Ihlen, Ø. (2007). Building on Bourdieu: A sociological grasp of public relations. Public Relations Review, 33(3), 269-274. DOI: 10.1016/j. pubrev.2007.05.005

Instituto Nacional de Estadística y Geografía (INEGI). (2016). Áreas geoestadisticas municipales. Aguascalientes, México: INEGI.

Lappe-Oliveras, P., Moreno-Terrazas, R., ArrizónGaviño, J., Herrera-Suárez, T., García-Mendoza, A. y Gschaedler-Mathis, A. (2008). Yeasts associated with the production of Mexican alcoholic nondistilled and distilled agave beverages. 
FEMS Yeast Research, 8(7), 1.037-1.052. DOI: 10.1111/j.1567-1364.2008.00430.x

Liang, J. y Chan, C. S. (2018). Local cultural vicissitudes in regional tourism development: A case of Zhuhai. Tourism Management Perspectives, 25, 80-92. DOI: $10.1016 /$ j.tmp.2017.11.016

Lutz, B. (2012). Civilizar al campesino pobre: biopolíticas alimentarias en México. RURISRevista do Centro de Estudos Rurais-UNICAMP, 6(2), 91-122. Recuperado de https://www.ifch. unicamp.br/ojs/index.php/ruris/article/view/1539

Marín, G., García, A. y Daltabuit, M. (coords.). (2012). Turismo, globalización y sociedades locales en la península de Yucatán, México (Colección PASOS Edita, n. ${ }^{\circ}$ 7). Tenerife: PASOS. Revista de Turismo y Patrimonio Cultural. Recuperado de http://www.pasosonline.org/Publicados/ pasosoedita/PSEdita7.pdf

Matta, R. (2016). Food incursions into global heritage: Peruvian cuisine's slippery road to Unesco. Social Anthropology, 24(3), 338-352. DOI: $10.1111 / 1469-8676.12300$

Matta, R. (2019). Celebrity chefs and the limits of playing politics from the kitchen. En J. Dürrschmidt y Y. Kautt (eds.), Globalized eating cultures (pp. 183-201). London: Palgrave Macmillan.

Mazzetto, E. y Moragas, N. (2015). Simbolismo y uso litúrgico de algunas variedades de octli entre los antiguos nahuas. Un primer acercamiento. Revista de Estudos da Religião (REVER), 15(1), 31-47. Recuperado de https://revistas.pucsp.br/ rever/article/view/23584

Mitchell, C. y Shannon, M. (2018). Exploring cultural heritage tourism in rural Newfoundland through the lens of the evolutionary economic geographer. Journal of Rural Studies, 59, 21-34. DOI: $10.1016 /$ j.jrurstud.2017.12.020

Moguillansky, M. (2008). Una genealogía del concepto de intermediario cultural. Revisiones recientes y aplicaciones en procesos de integración regional. Ponencia presentada en el IX Congreso Argentino de Antropología Social, Universidad Nacional de Misiones, Facultad de Humanidades y Ciencias Sociales, Posadas. Recuperada de https://www.aacademica. org/000-080/447

Parra, L. A., Del Villar, P. y Prieto, A. (2010). Extracción de fibras de agave para elaborar papel y artesanías. Acta Universitaria, 20(3), 77-83. Recuperado de https://www.redalyc.org/ pdf/416/41618860011.pdf

Piñuel, J. L. (2002). Epistemología, metodología y técnicas del análisis de contenido. Estudios de Sociolingüística, 3(1), 1-42. Recuperado de https://www.ucm.es/data/cont/docs/268-2013-0729-Pinuel_Raigada_AnalisisContenido_2002 EstudiosSociolinguisticaUVigo.pdf

Prats, L. (2005). Concepto y gestión del patrimonio local. Cuadernos de Antropología Social, 21, 17-35. Recuperado de https://www.redalyc.org/ pdf/1809/180913910002.pdf

Ramírez, R. (2004). El maguey y el pulque: memoria y tradición convertidos en historia, 1884-1993 (Tesis de licenciatura). Benemérita Universidad Autónoma de Puebla, Puebla (México).

Ramírez, R. (2007). La representación popular del maguey y el pulque en las artes. Cuicuilco, 14(39), 115-149. Recuperado de http://200.188.19.20/ islandora_74/islandora/object/articulo\%3A10413

Ramírez, I., Cruz, G., Vargas, E. y Rodríguez, I. (2019). Turismo en Teotihuacán y San Martín de Las Pirámides: su influencia en el capital social de los actores locales. Rosa dos Ventos - Turismo e Hospitalidade 11(4), 728-753. DOI: 10.18226/21789061.v11i4p728

Ramos-Elorduy, J. y Viejo Montesinos, J. L. (2007). Los insectos como alimento humano: breve ensayo sobre la entomofagia, con especial referencia a México. Boletín Real Sociedad 
Española de Historia Natural. Sección Biología, 102(1-4), 61-84. Recuperado de http://www. rsehn.es/index.php? $\mathrm{d}=$ publicaciones $\&$ num $=6 \&$ $\mathrm{w}=43 \& \mathrm{ft}=1$

Rendón, R. (1990). Dos haciendas pulqueras en Tlaxcala, 1857-1884. Tlaxcala: Gobierno del Estado de Tlaxcala y Universidad Iberoamericana.

Rivas, F. (2001). El maguey y el pulque en Teotihuacán: representación y simbolismo. Arqueología, 25, 47-62.

Rossi, A., Bui, S. y Marsden, T. (2019). Redefining power relations in agri-food systems. Journal of Rural Studies, 68, 147-158. DoI: 10.1016/j. jrurstud.2019.01.002

Schmitz, A., Witte, D. y Gengnagel, V. (2017). Pluralizing field analysis: Toward a relational understanding of the field of power. Social Science Information, 56(1), 49-73. DOI: $10.1177 / 0539018416675071$

Sieweke, J. (2014). Pierre Bourdieu in management and organization studies - A citation context analysis and discussion of contributions. Scandinavian Journal of Management, 30(4), 532-543. DOI: 10.1016/j.scaman.2014.04.004

Stake, R. (2000). Case studies. En N. Denzin y Y. Lincoln (eds.), Handbook of qualitative research (2. ${ }^{a}$ ed., pp. 435-454). London: Sage.
Suremain, C-É. (2017). Cuando la alimentación se hace patrimonio. Rutas gastronómicas, globalización y desarrollo local (México). Trace. Travaux et Recherches dans les Amériques du Centre, 72,165-181. DOI: 10.22134/trace.72.2017.114

Suremain, C-É. y Matta, R. (2013). 'Manger tradition' ou la fabrication d'un patrimoine alimentaire inégal. Trace. Travaux et Recherches dans les Amériques du Centre, 64, 44-54. DOI: 10.22134/trace.64.2013.64

Thomé-Ortiz, H., Renard-Hubert, M. C. y De Jésus Contreras, D. (2017). Turismo culinario y patrimonio histórico: la ruta de la sal prehispánica en Zapotitlán Salinas, México. Dos Algarves: A Multidisciplinary e-Journal, 30, 72-84. DOI: 10.18089/DAMeJ.2017.30.6

Unesco. (17 de octubre de 2003). Convención para la Salvaguardia del Patrimonio Cultural Inmaterial [En línea]. Recuperado de http://unesdoc. Unesco.org/images/0013/001325/132540s.pdf

Valadez, M. J. (2014). "Pulque limpio" / "pulque sucio": disputas en torno a la legitimidad y la producción social del valor. Revista Colombiana de Antropología, 50(2), 41-63. DOI: $10.22380 / 2539472 X 46$ 
